\title{
Editorial
}

\section{COVID-19 and liver: A critical relationship}

\author{
Eduardo Fernández-Martínez $^{a}$
}

The world faced a new challenge since the appearance of the severe acute respiratory syndrome coronavirus-2 (SARS-CoV-2) infection that led to the pandemic of coronavirus disease in 2019 (COVID-19). The lungs, heart, vasculature, kidneys, pancreas, brain, and liver are the most affected organs because they express the angiotensin-converting enzyme (ACE)-2, the host target receptor of SARS-CoV$2 .^{[1,2]}$ Indeed, the liver seems to be the second organ disturbed by COVID-19 because of its anatomical position and high blood supply by the hepatic artery and portal vein into the hepatic sinusoids; besides, the liver is recognized as a vital immunological organ due to its vast amount of phagocytic cells and fast immune response to infections. ${ }^{[2,3]}$ SARS-CoV-2 infection promotes an intense and sustained production and release of proinflammatory cytokines and chemokines, such as interleukin (IL)- $1 \beta$ and 6 , interferon (IFN)- $\gamma$, tumor necrosis factor (TNF)- $\alpha$, monocyte chemotactic protein (MCP)-1, among others; also, the anti-inflammatory IL-10. That abnormal increase of cytokines is known as the systemic cytokine storm, produced by the immune system cells, lymphocytes T and B, monocytes, macrophages, and cells of multiple infected organs. It is important to mention that patients with chronic immune-mediated illnesses may develop severe hyper-inflammatory complications. ${ }^{[1,4]}$ Therefore, many reports point out the COVID-19-induced liver damage (14-53\% of total patients), while the incidence of liver injured patients related with the illness degree of COVID-19 is $18 \%$ in nonsevere, $56 \%$ in severe, and 58-78\% in death. ${ }^{[5,6]}$ The SARS-CoV-2 infection causes direct cytopathic effects in the liver by mechanisms such as mitochondrial swelling and protein interactions, besides other hepatocyte organelles derangement. ${ }^{[7,8]}$ The presence of ACE-2 in hepatocytes might explain their vulnerability to the virus infection, but ACE-2 expression in hepatocytes is poor compared to the higher expression of this receptor in cholangiocytes. Although cholangiocytes also express the transmembrane serine protease (TMPRSS)-2, essential for the proteolytic activation and virus entry to cells, they do not develop significant damage. ${ }^{[7,9,10]}$ Another direct cause of COVID-19-induced liver injury is the cytokine storm that induces a hyper-proinflammatory state, causing cardiomyopathy before congestive hepatopathy, both characterized by ischemia, hypoxia, coagulopathy, as well as cellular necrosis and apoptosis; ${ }^{[6-10]}$ accordingly, these events are reinforced by liver immune reactivity and its high blood flow. Drug-induced liver damage (DILI) in COVID-19 patients can be another cause of hepatic injury during hospitalization because some of the medications administered to these patients have been reported as DILI inducers by hepatocellular, cholestatic, or mixed damage; for instance, acetaminophen (paracetamol), azithromycin, statins, hydroxychloroquine, lopinavir, ritonavir, remdesivir, favipiravir, tocilizumab, and baricitinib. ${ }^{[6-9]}$ COVID-19 may worsen underlying chronic liver diseases, given that 2-11\% of patients with COVID-19 suffer from chronic hepatitis B or C, alcoholic liver disease, non-alcoholic steatohepatitis, cirrhosis, hepatocellular carcinoma, and autoimmune liver diseases such as primary biliary cirrhosis, primary sclerosing cholangitis, or autoimmune hepatitis; indeed, these patients have a poorer prognosis since COVID-19 may provoke an acute decompensation and acute-on-chronic liver damage. ${ }^{[5-7,9,10]}$ The typical clinical findings in blood biochemical markers of liver damage in COVID-19 patients are a frequent rise from mild to moderate levels up to 5fold the upper limit of normal (ULN) of the necrosis indicators aspartate aminotransferase (AST) and alanine aminotransferase (ALT); often, their enzyme activities reach higher values in severe cases of COVID-19. The cholestasis markers are increased to a lesser extent since the gamma-glutamyl transferase (GGT) augments 3 x ULN, but increases in severe cases, alkaline phosphatase (ALP) is rarely abnormal, while total bilirubin (TB) concentration is elevated too. Albumin, an indicator of liver function, is diminished and even more 
in critical patients, then it has become a reliable predictor of mortality. ${ }^{[6-10]}$ Finally, liver damage by direct or indirect effects of SARSCoV-2 infection must be considered in clinics as very likely jeopardy; besides, patients should be managed consequently to the underlying comorbidities, modification of doses and treatments, age, sex, and degree of illness by COVID-19 to improve their prognosis and reduce their mortality.

\section{References}

[1] Tay MZ, Poh CM, Rénia L, MacAry PA, Ng LFP. The trinity of COVID-19: immunity, inflammation and intervention. Nat. Rev. Immunol. 2020; 20(6): 363-74. https://doi.org/10.1038/s41577-020-0311-8.

[2] Mokhtari T, Hassani F, Ghaffari N, Ebrahimi B, Yarahmadi A, Hassanzadeh G. COVID-19 and multiorgan failure: A narrative review on potential mechanisms. J. Mol. Histol. 2020; 51(6): 613-28. https://doi.org/10.1007/s10735-020-09915-3.

[3] Kubes P, Jenne C. Immune responses in the liver. Annu. Rev. Immunol. 2018; 36: 247-77. https://doi.org/10.1146/annurev-immunol-051116-052415.

[4] Schett G., Sticherling M, Neurath, MF. COVID-19: risk for cytokine targeting in chronic inflammatory diseases? Nat. Rev. Immunol. 2020; 20(5): 271-2. https://doi.org/10.1038/s41577-020-0312-7.

[5] Zhang C, Shi L, Wang FS. Liver injury in COVID-19: management and challenges. Lancet Gastroenterol. Hepatol. 2020; 5(5): 428-30. https://doi.org/10.1016/S2468-1253(20)30057-1.

[6] Amin M. COVID-19 and the liver: overview. Eur. J. Gastroenterol. Hepatol. 2020; 33(3): 309-11. https://doi.org/10.1097/MEG.0000000000001808.

[7] Metawea MI, Yousif WI, Moheb I. COVID 19 and liver: An A-Z literature review. Dig. Liver Dis. 2021; 53(2): 146-52. https://doi.org/10.1016/j.dld.2020.09.010

[8] Schaefer EAK, Arvind A, Bloom PP, Chung RT. Interrelationship between coronavirus infection and liver disease. Clin. Liver Dis. 2020 ; 15(5): 175-80. https://doi.org/10.1002/cld.967.

[9] Elhence A, Vaishnav M, Biswas S, Chauhan A, Anand A, Shalimar. Coronavirus disease-2019 (COVID-19) and the liver. J. Clin. Transl. Hepatol. 2021; 9(2): 247-55. https://doi.org/10.14218/JCTH.2021.00006.

[10] Jothimani D, Venugopal R, Abedin MF, Kaliamoorthy I, Rela M. COVID-19 and the liver. J.Hepatol. 2020; 73(5): 1231-40. https://doi.org/10.1016/j.jhep.2020.06.006. 